\section{Associação entre nível de atividade física nós diferentes domínios e sintomas do envelhecimento masculino}

\author{
Association between physical activity \\ level in different domains and aging \\ males' symptoms
}

Leandro Quadro Corrêa $a^{1,3}$

Marcelo Cozzensa da Silva ${ }^{2,3}$

Airton José Rombaldi ${ }^{2,3}$

\section{RESUMO}

O objetivo deste estudo foi verificar a associação dos escores geral e parciais (psicológicos, somáticos e sexuais) dos sintomas do envelhecimento masculino com o nível de atividade física (AF) nos diferentes domínios (doméstico, trabalho, deslocamento e lazer). Foi realizado estudo transversal de base populacional, com amostra representativa de homens de 40 anos de idade ou mais residentes na zona urbana de Pelotas-RS ( $\mathrm{n}=421)$. Para avaliar os sintomas do envelhecimento masculino foi utilizada a escala (AMS) e para verificar o nível de AF nos diferentes domínios foi utilizado o (IPAQ), versão longa. Foram considerados ativos aqueles que realizaram ao menos 150 minutos por semana de $\mathrm{AF}$ e com sintomas do envelhecimento aqueles com escore igual ou superior a 37 pontos na escala AMS. O escore geral dos sintomas do envelhecimento masculino esteve significativa e inversamente associado com o nível de AF nos domínios do lazer $(\mathrm{p}=0,04)$ e doméstico $(\mathrm{p}=0,02)$. Adicionalmente, $\mathrm{o}$ escore dos sintomas somáticos do envelhecimento esteve associado com o nível de $\mathrm{AF}$ no lazer $(\mathrm{p}=0,02)$. Concluiu-se que que homens mais ativos fisicamente no lazer e no domicílio tendem a apresentar menores sintomas do envelhecimento, bem como os ativos no lazer apresentam menores sintomas somáticos. A prática regular de $\mathrm{AF}$ deve ser incentivada em homens com mais de 40 anos, pois a inatividade física potencializa o processo de envelhecimento.

\section{PALAVRAS-CHAVE}

Envelhecimento; Homens; Atividade motora.

\begin{abstract}
The aim of this study was to investigate the association between the whole and the partial scores (psychological, somatic and sexual) of aging males' symptoms and physical activity level (PA) in different domains assessed separately (at home, at work, commuting and at leisure-time). This population -based cross-sectional study was conducted in a representative sample of men aged 40 years or older living in the urban area of Pelotas, southern Brazil ( $n=421)$. The Aging Males' Symptoms Scale (AMS) was used to assess aging males' symptoms and the International Physical Activity Questionnaire (IPAQ), long version, was used to verify the level of PA. Those who performed at least $150 \mathrm{~min} /$ week of $P A$ were considered active and those with a score $\geq 37$ points on the AMS scale were considered positive to aging symptoms. The whole aging males' symptoms score was inversely associated with the level of PA in leisure-time and domestic activities ( $p=0.04$ and $p=0.02$, respectively); insufficiently active males showed more aging symptoms in comparison with the active. The somatic symptoms were also associated with the PA level in the leisure-time domain, where the insufficiently active males in this domain presented more symptoms in comparison to the active $(p=0.02)$. It was concluded that physically active men in leisure-time and domestic activities tend to have lower aging symptoms, as well as active men in leisure time have lower somatic symptoms. Systematic practice of PA should be encouraged for men aged 40 years or older as physical inactivity enhances the aging process.
\end{abstract}

\section{KEYWORDS}

Aging; Men; Motor activity.
Rev Bras Ativ Fis Saúde p. 186-194 DOI

http://dx.doi.org/10.12820/rbafs.v.19n2p186

1 Curso de Educação Física - Universidade Federal do Rio Grande, Rio Grande, Brasil

2 Programa de Pós-graduação em Educação Física, Universidade Federal de Pelotas, Pelotas, Brasil

3 Grupo de Estudos em Epidemiologia da Atividade Física (GEEAF), Universidade Federal de Pelotas, Pelotas, Brasil 


\section{INTRODUÇÃO}

O envelhecimento populacional é um fenômeno global e vem ocorrendo mais rapidamente em países pobres e em desenvolvimento ${ }^{1}$ em consequência da redução nas taxas de fecundidade e de mortalidade infantil e do aumento da expectativa de vida ${ }^{1}$. Doenças crônicas não transmissíveis (DCNT) como as doenças cardíacas, derrame, perdas visual e auditiva e demência estão entre os maiores desafios à saúde da população mais velha ${ }^{1}$. No entanto, essas condições patológicas podem ter seus efeitos minimizados pela adoção de um estilo de vida saudável que inclua a prática regular de atividade física $(\mathrm{AF})^{1,2}$.

Os sintomas do envelhecimento caracterizam-se por um conjunto de fatores de ordem psicológica, somática e sexual que podem influenciar negativamente a saúde de homens mais velhos ${ }^{3}$. Nesse sentido, estudos sobre o envelhecimento masculino foram conduzidos a partir do final da década de $1990^{4-7}$ para determinar a prevalência dos sintomas a ele relacionados e reportaram prevalências elevadas, tanto em países desenvolvidos ${ }^{4,5}$ como em desenvolvimento $^{6,7}$.

Atualmente, a inatividade física tem sido considerada um fator de risco ou conduta de risco ${ }^{8}$, sendo uma das causas evitáveis pelo aumento do risco de adoecimento por DCNT na população idosa ${ }^{1}$. Estudos prévios ${ }^{7,9}$ conduzidos em uma população do Sul do Brasil, relataram que os sintomas do envelhecimento masculino estavam associados a inatividade física, sendo que a prevalência e a severidade dos sintomas foram maiores entre homens insuficientemente ativos quando comparado aos fisicamente ativos, seja quando avaliada a $\mathrm{AF}$ total ou nos domínios de lazer e deslocamento conjuntamente. Entretanto, até o presente momento, nenhum estudo verificou a associação entre os escores parciais dos sintomas do envelhecimento masculino e os níveis de atividade física em diferentes domínios (doméstico, trabalho, deslocamento e lazer).

O presente estudo teve como objetivo verificar a associação entre o nível de atividade física nos diferentes domínios (doméstico, trabalho, deslocamento e lazer) com os escores dos sintomas do envelhecimento masculino (psicológicos, somáticos e sexuais) em indivíduos, com idade igual ou superior a 40 anos de idade, residentes na zona urbana do município de Pelotas.

\section{MÉTODOS}

Este estudo, caracterizado como transversal e de base populacional, foi realizado no segundo semestre de 2007, no município de Pelotas. Esta cidade do extremo sul do Rio Grande do Sul tem aproximadamente 340.000 habitantes, sendo que desses, aproximadamente 112.000 (32\% da população) apresentavam idade igual ou superior a 40 anos $^{10}$. O Instituto Brasileiro de Geografia e Estatística (IBGE) divide o município em 404 setores censitários urbanos dos quais foram sorteados 45 para serem incluídos no estudo. Em cada setor censitário sorteado foi determinado o ponto de partida do estudo, a partir do qual foram selecionados sistematicamente os domicílios a serem visitados.

Após a seleção do primeiro domicílio a ser incluído no estudo, os próximos foram selecionados de forma sistemática, respeitando-se o intervalo estipulado de cinco domicílios, até atingir as 20 residências em cada setor. No total foram selecionadas 900 residências onde todos os homens que apresentassem 
idade igual ou superior a 40 anos foram inicialmente considerados elegíveis para o estudo. Foram excluídos do estudo indivíduos institucionalizados (asilos, hospitais, prisões e quartéis), indivíduos com incapacidade motora severa (tetraplégicos, paralisia cerebral, entre outras) e indivíduos que não tivessem capacidade de responder e/ou compreender as perguntas do questionário.

Para o cálculo do tamanho amostral, utilizou-se uma estimativa de prevalência de $20 \%$ de sintomas do envelhecimento masculino para homens de 40 anos ou mais, um erro aceitável de quatro pontos percentuais e nível de significância de $95 \%$. O tamanho amostral calculado inicialmente foi de 384 homens. Foi acrescido a esse valor $10 \%$ para perdas e recusas, sendo o "n" final de 422 sujeitos com idade de 40 anos ou mais.

Para verificar a prevalência dos sintomas do envelhecimento masculino utilizou-se a escala The Aging Males' Simptoms Scale (AMS), composta por 17 questões divididas em três blocos: sintomas psicológicos (cinco questões), somáticos (sete questões) e sexuais (cinco questões). O resultado de cada uma das questões pode variar de zero (melhor condição) a cinco pontos (pior condição) e o somatório geral de pontos das questões indica a pontuação final obtida para cada indivíduo. No presente estudo, trabalhou-se com os escores parciais (representado pelo escore de cada um dos sintomas: psicológico, somático e sexual) e com um escore de sintoma geral do envelhecimento, o qual representava o somatório da pontuação obtida por todos escores parciais ${ }^{4}$.

Posteriormente, o escore geral dos sintomas de envelhecimento foi dicotomizado de modo que aqueles que apresentaram valores $\geq 37$ pontos foram classificados como tendo sintomas do envelhecimento, enquanto aqueles com valores inferiores a este escore foram apontados como sem sintomas ${ }^{5}$. Os escores parciais dos sintomas psicológicos, somáticos e sexuais também foram dicotomizados, sendo classificados como tendo sintomas psicológicos os sujeitos que tiveram medida $\geq 12$ pontos; como tendo sintomas somáticos, os sujeitos que alcançaram valor $\geq 13$ pontos e, com sintomas sexuais, aqueles sujeitos que apresentaram valores $\geq 8$ pontos 9 .

Para quantificar o nível de $\mathrm{AF}$ dos homens nos quatro domínios, utilizouse a versão longa do International Physical Activity Questionnaire (IPAQ). Foram considerados ativos aqueles que atingiram, pelo menos, $150 \mathrm{~min} / \mathrm{sem}$ de atividades físicas (em cada um dos domínios isoladamente), de acordo com as recomendações do American College of Sports Medicine e World Health Organization $^{11,12}$.

Além disso, foi utilizado um questionário padronizado para descrever algumas características dos sujeitos. Os dados coletados foram idade (em anos completos), cor da pele (categorizados em brancos, não brancos, conforme percepção do entrevistador); situação conjugal (com companheiro, sem companheiro); classe econômica (ABEP 2008 - A, B, C, D/E) ${ }^{13}$; escolaridade (em anos completos de estudo); tabagismo (fumante atual, ex-fumante, nunca fumou); índice de massa corporal [IMC] (normal; sobrepeso, obesidade), e; autopercepção de saúde (excelente, muito boa, boa, regular, ruim). O questionário foi testado em um estudo piloto realizado em um setor censitário não incluído na amostra final.

Ambos os instrumentos foram aplicados face-a-face, com exceção do bloco dos sintomas sexuais que foi autoaplicado de forma a garantir o sigilo das informações, não expor os respondentes a situações de constrangimento e mini- 
mizar recusas ao estudo (homens que responderam ao questionário recebiam um envelope com as questões e, logo após responderem o mesmo, tinham seu documento lacrado). Aqueles homens que não tivessem condições de ler ou compreender as questões poderiam solicitar a leitura das mesmas pelos entrevistadores.

Os questionários foram aplicados por entrevistadores de ambos os sexos com, pelo menos, ensino médio completo, que receberam treinamento de 40 horas para aplicação do instrumento sem estarem informados dos objetivos nem das hipóteses do estudo. Os entrevistadores realizaram as entrevistas individualmente. Os supervisores do trabalho de campo refizeram as entrevistas em $10 \%$ da amostra, selecionada ao acaso, com um questionário reduzido, contendo questões chave selecionadas do instrumento para controle de qualidade do estudo.

O banco de dados foi construído no programa Epi Info 6.0, sendo realizada dupla digitação de cada questionário. Para análise dos dados utilizou-se o programa STATA 9.0. Foi feita uma análise descritiva dos sujeitos da amostra em termos dos sintomas do envelhecimento masculino, do nível de AF e das variáveis socioeconômicas, demográficas, comportamentais e de saúde. Para comparar a prevalência do nível de AF segundo os sintomas do envelhecimento masculino foram empregados o teste de Qui-quadrado e o teste Exato de Fisher. O nível de significância estabelecido foi $\mathrm{p}<0,05$.

Esse estudo foi aprovado pelo Comitê de Ética em Pesquisa da Escola Superior de Educação Física da Universidade Federal de Pelotas (protocolo 005/2008) e os dados foram coletados após consentimento informado dos sujeitos por escrito.

\section{RESULTADOS}

Dos 459 indivíduos com idade de 40 anos ou mais encontrados nos 876 domicílios selecionados, 421 foram efetivamente entrevistados (8,3\% de perdas e recusas, sendo a falta de tempo o principal motivo alegado para a recusa). $\mathrm{O}$ efeito de delineamento encontrado $(0,7)$ foi suficiente para manter o poder e o nível de confiança previstos pelo estudo.

A Tabela 1 apresenta a descrição da amostra, conforme variáveis sociodemográficas, nutricional e comportamentais. A média de idade dos homens investigados foi de $54,5 \pm 10,5$ anos; sendo que a maioria pertencia as classes econômicas mais baixas $(58,1 \%)$, tinham até oito anos ou menos de estudo (64,6\%); apresentava sobrepeso/obesidade (67,1\%); e menos de um quarto percebia sua saúde como excelente ou muito boa $(23,6 \%)$.

Verificou-se que 20,0\% (IC95\%: 16,14-24,31) apresentavam pontuação $\geq 37$ pontos na escala adotada para avaliação dos sintomas do envelhecimento masculino, condizentes com a presença deste tipo de sintoma. Já em relação aos diferentes tipos de sintomas que compõem o escore geral, 42,2\% (IC95\%: 37,3 - 47,4) dos homens apresentaram sintomas psicológicos, 35,9\% (IC95\%: 31,1 - 40,9) apresentaram sintomas somáticos e 64,4\% (IC95\%: 59,4-69,1) apresentaram sintomas sexuais (dados não apresentados nas tabelas).

Em relação ao nível de AF dos entrevistados, pode-se observar que poucos foram classificados como ativos nos domínios do deslocamento (3,3\% IC95\%: 1,8 - 5,5) e lazer (9,3\% - IC95\%: 6,7-12,4) (Figura 1). A Figura 2 
TABELA 1 - Características da amostra conforme variáveis sociodemográficas, nutricional, comportamental e de saúde de homens com 40 anos ou mais de idade da cidade Pelotas-RS, 2007 (n=421).

\begin{tabular}{|c|c|c|}
\hline Variáveis & $\mathrm{n}$ & $\%$ \\
\hline \multicolumn{3}{|l|}{ Faixa etária (anos) } \\
\hline $40-49$ & 164 & 39,0 \\
\hline $50-59$ & 137 & 32,6 \\
\hline $60-69$ & 77 & 18,3 \\
\hline$\geqslant 70$ & 43 & 10,1 \\
\hline \multicolumn{3}{|l|}{ Cor da pele } \\
\hline Branco & 357 & 85,2 \\
\hline Não branco & 62 & 14,8 \\
\hline \multicolumn{3}{|l|}{ Classe econômica } \\
\hline A (mais alta) & 33 & 8,0 \\
\hline $\mathrm{B}$ & 140 & 33,9 \\
\hline C & 190 & 46,2 \\
\hline D/E (mais baixa) & 49 & 11,9 \\
\hline \multicolumn{3}{|l|}{ Escolaridade (anos completos) } \\
\hline 0 & 14 & 3,3 \\
\hline 1 a 4 & 112 & 26,6 \\
\hline 5 a 8 & 146 & 34,7 \\
\hline 9 a 11 & 76 & 18,1 \\
\hline$\geqslant 12$ & 73 & 17,3 \\
\hline \multicolumn{3}{|l|}{ Situação conjugal } \\
\hline Casado ou morando com companheira & 325 & 77,2 \\
\hline Sem companheira & 96 & 22,8 \\
\hline \multicolumn{3}{|l|}{ IMCV $\left(\mathrm{kg} / \mathrm{m}^{2}\right)$} \\
\hline Normal & 133 & 32,9 \\
\hline Sobrepeso & 183 & 45,3 \\
\hline Obesidade & 88 & 21,8 \\
\hline \multicolumn{3}{|l|}{ Tabagismo } \\
\hline Nunca fumou & 128 & 30,4 \\
\hline Ex-fumante & 175 & 41,6 \\
\hline Fumante atual & 118 & 28,0 \\
\hline \multicolumn{3}{|l|}{ Autopercepção de saúde } \\
\hline Excelente & 41 & 9,8 \\
\hline Muito boa & 58 & 13,8 \\
\hline Boa & 216 & 51,4 \\
\hline Regular & 85 & 20,2 \\
\hline Ruim & 20 & 4,8 \\
\hline
\end{tabular}

IMC = índice massa corporal

relata a prevalência de indivíduos com sintomas de envelhecimento de acordo com o nível de AF nos diferentes domínios. Verificou-se que houve diferenças somente nos que atenderam e não atenderam a atual recomendação de AF nos domínios do lazer $(\mathrm{p}=0,04)$ e doméstico $(\mathrm{p}=0,02)$.

A Tabela 2 apresenta a associação entre os sintomas psicológicos, somáticos e sexuais do envelhecimento masculino com os diferentes domínios da AF. Verificou-se que o nível de AF no lazer esteve inversamente associada ao escore dos sintomas somáticos $(\mathrm{p}=0,02)$. 


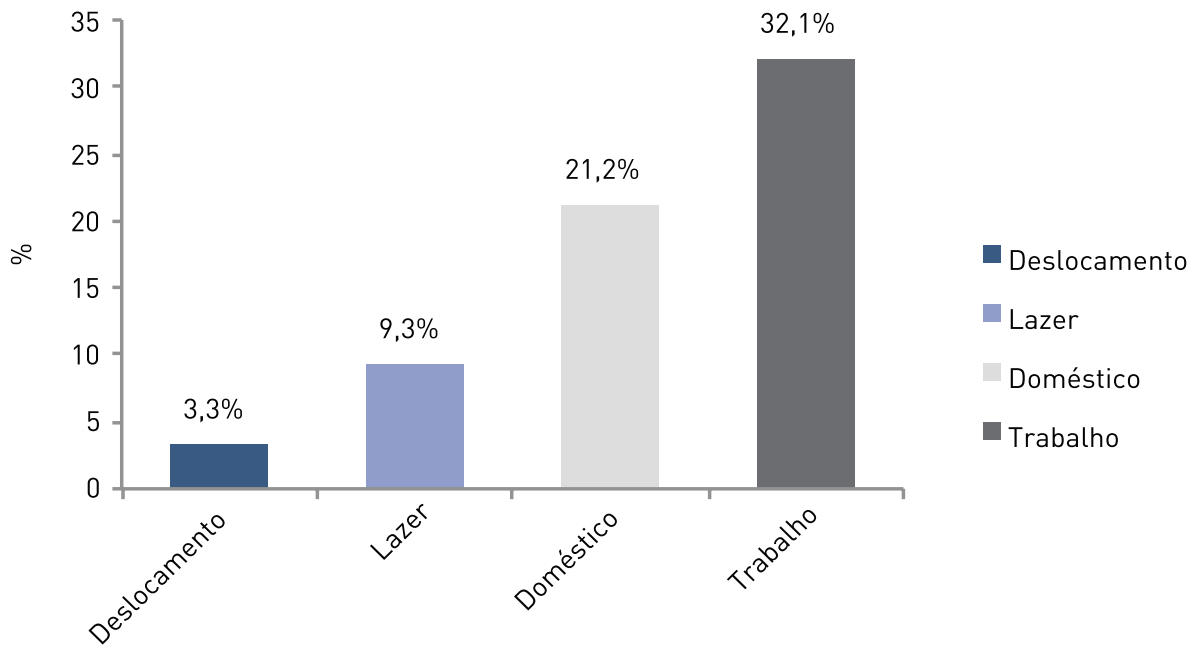

FIGURA 1 - Prevalência de prática suficiente de atividade física nos diferentes domínios (n=421).

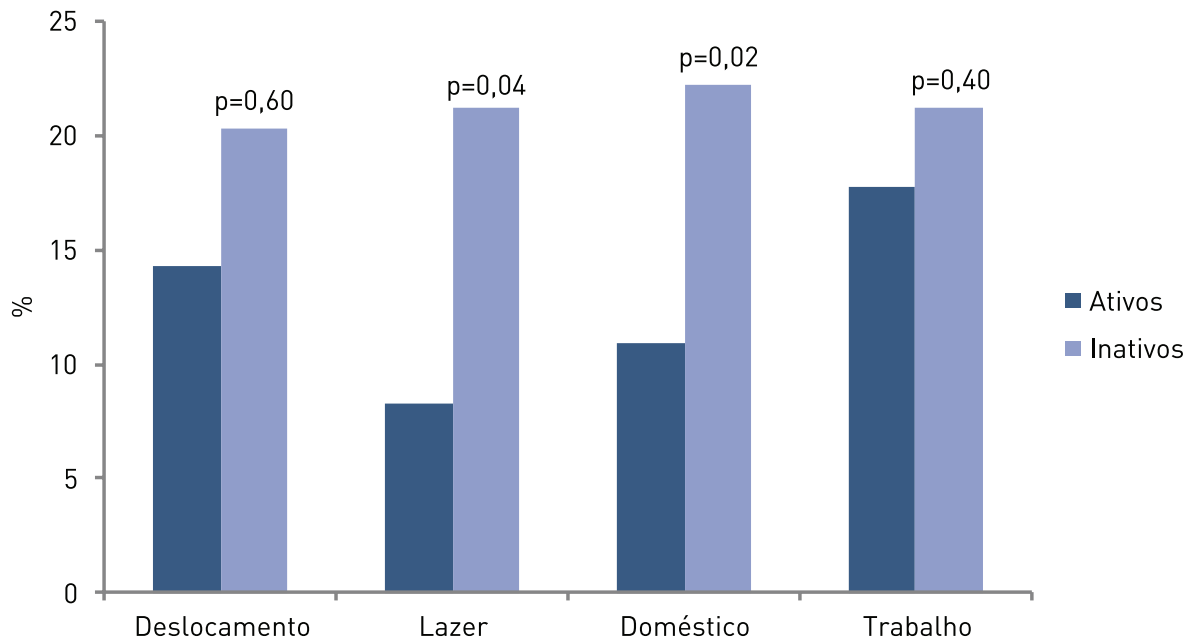

FIGURA 2 - Prevalência de indivíduos com sintomas do envelhecimento masculino nos diferentes domínios da atividade física ( $\mathrm{n}=421)$.

TABELA 2 - Associação entre os tipos de sintomas do envelhecimento masculino e o nível de atividade física nos diferentes domínios (n=421).

\begin{tabular}{|c|c|c|c|c|c|c|}
\hline & \multicolumn{2}{|c|}{ Sintomas psicológicos } & \multicolumn{2}{|c|}{ Sintomas somáticos } & \multicolumn{2}{|c|}{ Sintomas sexuais } \\
\hline & $\operatorname{Sim}[n(\%)]$ & Não [n (\%)] & $\operatorname{Sim}[n(\%)]$ & Não [n (\%)] & $\operatorname{Sim}[n(\%)]$ & Não [n (\%)] \\
\hline Atividade física - lazer & \multicolumn{2}{|c|}{$p=0,08^{*}$} & \multicolumn{2}{|c|}{$p=0,02^{*}$} & \multicolumn{2}{|c|}{$p=0,10^{*}$} \\
\hline Ativos & $213(56,5)$ & $27(71,0)$ & $235(62,3)$ & $31(81,6)$ & $122(34,5)$ & $17(47,2)$ \\
\hline Insuficientemente ativos & $164(43,5)$ & $11(29,0)$ & $142(37,7)$ & $7(18,4)$ & $232(65,5)$ & $19(52,8)$ \\
\hline Atividade física - trabalho & \multicolumn{2}{|c|}{$p=0,70^{*}$} & \multicolumn{2}{|c|}{$p=0,70^{*}$} & \multicolumn{2}{|c|}{$p=0,10^{*}$} \\
\hline Ativos & $163(58,4)$ & $76(56,3)$ & $177(63,4)$ & $88(65,2)$ & $85(32,7)$ & $53(41,1)$ \\
\hline Insuficientemente ativos & $116(41,6)$ & $59(43,7)$ & $102(36,6)$ & $47(34,8)$ & $175(67,3)$ & $76(58,9)$ \\
\hline Atividade física - deslocamento & \multicolumn{2}{|c|}{$p=0,20^{\#}$} & \multicolumn{2}{|c|}{$p=0,40^{\#}$} & \multicolumn{2}{|c|}{$p=0,50^{\#}$} \\
\hline Ativos & $229(57,3)$ & $10(71,4)$ & $254(63,5)$ & $11(78,6)$ & $134(35,7)$ & $4(28,6)$ \\
\hline Insuficientemente ativos & $171(42,7)$ & $4(28,6)$ & $146(36,5)$ & $3(21,4)$ & $241(64,3)$ & $10(71,4)$ \\
\hline Atividade física - domicílio & \multicolumn{2}{|c|}{$p=0,30^{*}$} & \multicolumn{2}{|c|}{$p=0,05^{*}$} & \multicolumn{2}{|c|}{$p=0,70^{*}$} \\
\hline Ativos & $186(56,7)$ & $54(62,8)$ & $203(61,9)$ & $63(73,3)$ & $108(35,2)$ & $31(37,8)$ \\
\hline Insuficientemente ativos & $142(43,3)$ & $32(37,2)$ & $125(38,1)$ & $23(26,7)$ & $199(64,8)$ & $51(62,2)$ \\
\hline
\end{tabular}

*Teste de Qui-quadrado; \#Teste Exato de Fisher 


\section{DISCUSSÃO}

O presente estudo objetivou verificar a associação entre o nível de atividade física nos diferentes domínios (doméstico, trabalho, deslocamento e lazer) com os escores dos sintomas do envelhecimento masculino (psicológicos, somáticos e sexuais).

Os resultados desse estudo revelaram que o nível de AF no lazer foi estatisticamente associado ao escore geral dos sintomas de envelhecimento masculino, sendo que os homens que não atenderam as recomendações mínimas de $\mathrm{AF}$ apresentaram maior prevalência desses sintomas em comparação àqueles que atingiram ou faziam mais que o recomendado. Independentemente do escore (parcial ou total), a percepção do envelhecimento se mostrou inversamente proporcional a quantidade de prática de AF realizada no lazer. Atividades físicas realizadas no tempo de lazer, normalmente são executadas de forma espontânea e prazerosas, o que pode produzir um conjunto de efeitos fisiológicos e psicológicos que implicam na modificação da percepção ${ }^{8}$.

A prevalência de AF no domínio doméstico foi de, aproximadamente, 21,0\%. Estudo recentemente publicado por Del Duca e colegas ${ }^{15}$ em uma amostra de homens brasileiros com idades entre 20 e 59 anos, encontrou uma prevalência de AF semelhante a esta pesquisa. No que diz respeito a prática de atividades físicas no ambiente doméstico, parece haver uma tendência a superestimativa de relato das atividades deste domínio entre os indivíduos, independente do sexo.

A prevalência de $\mathrm{AF}$ no domínio do lazer foi menor que a encontrada em estudo de Del Duca e colaboradores ${ }^{16}$ onde as frequências encontradas foram de $45,2 \%$ e 43,4\%, respectivamente, para as idades entre 40 a 49 anos e 50 e 59 anos. Em estudo realizado na cidade de Pelotas, Dias da Costa et al. encontrou prevalência de $\mathrm{AF}$ no lazer de $17,1 \%$ em indivíduos com idade entre 45 e 54 anos e de $11,8 \%$ entre aqueles com idade entre 55 e 69 anos $^{17}$. Estudo de Feeny e colaboradores ${ }^{18}$ demonstrou que a $\mathrm{AF}$ realizada no tempo de lazer exerce, independentemente do índice de massa corporal dos indivíduos, forte influência sobre a qualidade de vida dos sujeitos. Apesar disso, é bem reconhecido que com o avanço da idade, decresce a prevalência de $\mathrm{AF}$ no lazer entre os indivíduos ${ }^{16,17}$. Esse avanço da idade já foi reconhecidamente associado com barreiras à AF tais como falta de dinheiro, falta de companhia, falta de tempo, sentir-se cansado, medo de lesões, não gostar de realizar exercícios, sentir-se muito velho, entre ou$\operatorname{tros}^{19}$. Estudos prévios com essa mesma população também demonstraram menores prevalências de AF entre indivíduos com sintomas do envelhecimento ${ }^{7,9}$.

Os valores achados vão ao encontro de um estudo prévio em que os autores encontraram associações entre os sintomas somáticos, além dos psicológicos e do escore geral do envelhecimento, com o nível insuficiente de AF. Entretanto, esse estudo avaliou a AF nos domínios do lazer e deslocamento de forma conjunta ${ }^{6}$.

No que diz respeito aos sintomas somáticos do envelhecimento masculino, estudos tem apontado que a prática regular de AF é capaz de combater a perda de força e de massa muscular, minimizando o risco de fraturas e o comprometimento da qualidade de vida das pessoas nessa faixa etária ${ }^{20,21}$. Além disso, a AF contribui para o aumento da aptidão cardiorrespiratória ${ }^{20,22}$ e da densidade mineral óssea ${ }^{23}$, melhora o perfil lipídico ${ }^{24}$ e reduz o percentual de gordura e seus marcadores ${ }^{25}$, ajuda na redução da insônia ${ }^{26}$ e das dores ${ }^{27,28}$, os quais são características somáticas do envelhecimento. 
Desse modo, ressaltamos a importância da prática de AF para a população como um todo, pelo impacto à saúde que esse comportamento é capaz de proporcionar. No caso dos idosos, ressalta-se a importância da AF no domínio doméstico, pois o tempo de permanência no domicílio entre essas pessoas é grande e as AF realizadas nesse ambiente, mesmo que de caráter leve a moderado, podem contribuir para a melhoria da saúde física e mental.

Deve-se ressaltar que este foi o primeiro estudo a avaliar a associação entre o escore geral e os escores parciais dos sintomas do envelhecimento masculino com os diferentes domínios da AF. Entretanto, não se pode descartar a possibilidade de ocorrência de causalidade reversa entre o desfecho estudado e a prática de atividades físicas nos diferentes domínios. Além disso, é importante salientar que alguns indivíduos podem ter sido mal classificados no domínio de trabalho medido pelo IPAQ (superestimação do tempo ativo no trabalho), o que pode ter levado a uma aproximação entre os percentuais do escore geral dos sintomas do envelhecimento, o que levaria a uma diluição da associação.

Verificou-se que o nível de AF no lazer esteve inversamente associado aos sintomas gerais do envelhecimento masculino e aos sintomas somáticos. Tendo em vista que esses sintomas podem trazer consequências negativas nos aspectos psicológicos, somáticos e sexuais nos homens em processo de envelhecimento, faz-se necessário a implementação e o estímulo de políticas que contribuam para que a população mais velha modifique seu comportamento no que diz respeito a prática de $\mathrm{AF}$, tornando-se mais ativa, de modo a minimizar os efeitos negativos proveniente do estilo de vida inativo.

\section{Contribuição dos autores}

LQC idealizou o estudo e liderou a escrita do artigo e a análise dos dados. MCS participou da escrita do artigo e da análise dos dados. AJR participou de todas as etapas de construção deste trabalho.

\section{REFERÊNCIAS}

1. World Health Organization. Good health adds life to years. Global brief for World Health Day 2012. World Health Organization, Geneve: 2012. [citado 2013 mar 20]. Disponível em: http://whqlibdoc.who.int/hq/2012/WHO_DCO_WHD_2012.2_eng.pdf.

2. Brasil, Ministério da Saúde. Envelhecimento e saúde da pessoa idosa, Brasília: 2007. [citado 2013 mar 20]. Disponível em: http://bvsms.saude.gov.br/bvs/publicacoes/abcad19.pdf.

3. T'sjoen G, Goemaere S, De Meyere M, Kaufman JM. Perception of males' aging symptoms, health and well-being in elderly community-dwelling men is not related to circulating androgen levels. Psychoneuroendocrinology. 2004; 29(2): 201-14.

4. Heinemann LAJ, Zimmermann T, Vermeulen A, Thiel C, Hummel W. A new "aging males' symptoms" (AMS) rating scale. Aging Male. 1999; 2(2):105-14.

5. Ichioka K, Nishiyama H, Yoshimura K, Itoh N, Okubo K, Terai A. Aging males' symptoms scale in Japanese men attending a multiphasic health screening clinic. Urology. 2006;67(3):589-93.

6. Akinyemi A, Bamiwuye O, Inathaniel T, Ijadunola K, Fatusi A. The Nigerian Aging Males' Symptoms scale. Experience in elderly males. Aging Male. 2008; 11(2):89-93.

7. Corrêa LQ, Rombaldi AJ, Silva MC, Domingues MR. Aging male's symptoms in a Southern Brazil population: lifestyle effects after the age of 40. Aging Male. 2010; 13(2): 93-9.

8. Lee IM, Shiroma EJ, Lobelo F, Puska P, Blair SN, Katzmarzyk PT, et al. Effect of physical inactivity on major non-communicable diseases worldwide: an analysis of burden of disease and life expectancy. Lancet. 2012; 380(9838): 219-29.

9. Corrêa LQ, Rombaldi AJ, Silva MC. Atividade física e sintomas do envelhecimento masculino em uma população do Sul do Brasil. Rev Bras Med Esporte. 2011; 17(4):228-31. 
10. IBGE. Censo demográfico, 2000. [citado 2008 fev 20] Disponível em: http://www.ibge. gov.br.

11. Haskell WL, Lee IM, Pate RR, Powell KE, Blair SN, Franklin BA, et al. Physical activity and public health: updated recommendation for adults from the American College of Sports Medicine and the American Heart Association. Med Sci Sports Exerc. 2007; 39(8):1423-34.

12. World Health Organization. Global Recommendations on physical activity for health. World Health Organization, Geneva, 2010. [citado 2013 mar 20]. Disponível em: http://whqlibdoc.who.int/publications/2010/9789241599979_eng.pdf.

13. Associação Brasileira das Empresas de Pesquisa. Título. [citado 2008 Set 25]. Disponível: <http://www.abep.org>.

14. US Department of Health and Human Service. 2008 Physical activity guidelines for Americans. [citado 2013 mai 07]. Disponível em: <http://www.health.gov/paguidelines/ pdf/paguide.pdf $>$.

15. Del Duca GF, Nahas MV, de Sousa TF, Mota J, Hallal PC, Peres KG. Clustering of physical inactivity in leisure, work, commuting and household domains among Brazilian adults. Public Health. 2013; 127(6): 530-7.

16. Del Duca GF, Nahas MV, Garcia LMT, Mota J, Hallal PC, Peres MA. Prevalence and sociodemographic correlates of all domains of physical activity in Brazilian adults. Prev Med.2013; 56 (2): 99-102.

17. Dias-da-Costa JS, Hallal PC, Wells JC, Daltoé T, Fuchs SC, Menezes AM, et al. Epidemiology of leisure-time physical activity: a population-based study in southern Brazil. Cad Saude Publica. 2005;21(1):275-82.

18. Feeny D, Garner R, Bernier J, Thompson A, McFarland BH, Huguet N, et al. Physical activity matters: associations among body mass index, physical activity and healthrelated quality of life trajectories over 10 years. J Phys Act Health. 2013 Oct 31. [Epub ahead of print]

19. Reichert FF, Barros AJ, Domingues MR, Hallal PC. The role of perceived personal barriers to engagement in leisure-time physical activity. Am J Public Health. 2007;97(3):515-9.

20. Nóbrega ACL, Freitas EV, Oliveira MAB, Leitão MB, Lazzoli JK, Nahas RM, et al. Posicionamento oficial da Sociedade Brasileira de Medicina do Esporte e da Sociedade Brasileira de Geriatria e Gerontologia: atividade física e saúde no idoso. Rev Bras Med Esp. 1999; 5(6):207-11.

21. Deschenes MR. Effects of aging on muscle fibre type and size. Sports Med. 2004; 34(12):809-24.

22. Mazzeo R, Cavanagh P, Evans WJ, Fiatarone M, Hagberg J, Mcauley E, et al. Exercise and physical activity for older adults. Med Sci Sports Exerc. 1998; 30(6):992-1008.

23. Remes T, Väisänem SB, Mahonen A, Huuskonen J, Kröger H, Jurvelin JS, et al. The association of bone metabolism with bone mineral density, serum sex hormone concentrations, and regular exercise in middle-aged men. Bone. 2004; 35(2):439-47.

24. Rauramaa R, Tuomainen P, Väisänen S, Rankinen T. Physical activity and healthrelated fitness in middle-aged men. Med Sci Sports Exerc. 1995; 27(5):707-12.

25. Wagner A, Simon C, Ducimetière P, Montaye M, Bongard V, Yarnell J, et al. Leisuretime physical activity and regular walking or cycling to work are associated with adiposity and 5 y weight gain in middle-aged men: the PRIME Study. Int J Obes Relat Metab Disord. 2001; 25(7):940-8.

26. Inoue S, Yorifuji T, Sugiyama M, Ohta T, Ishikawa-Takata K, Doi H. Does habitual physical activity prevent insomnia? A cross-sectional and longitudinal study of elderly Japanese. J Aging Phys Act. 2013;21(2):119-39.

27. Bruce B, Fries JF, Lubeck DP. Aerobic exercise and its impact on musculoskeletal pain in older adults: a 14 year prospective, longitudinal study. Arthritis Res Ther. 2005; 7(6) R1263-70.

28. Pedrinelli A, Garcez-Leme LE, Nobre RSA. The effect of physical training on locomotive apparatus in elderly people. Rev Bras Ortop. 2009;44(2):96-101.

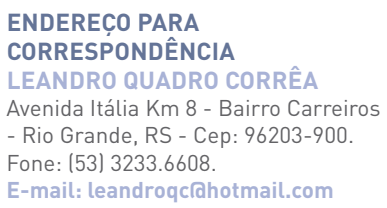

